\title{
sciendo
}

\section{Strength Profile of Hip Abductor and Adductor Muscles in Youth Elite Soccer Players}

\author{
by \\ Konstantina Karatrantou ${ }^{1}$, Vassilis Gerodimos ${ }^{1}$, Eleftheria Katsareli², \\ Nikolaos Manouras ${ }^{1}$, Panagiotis Ioakimidis ${ }^{1}$, Konstantinos Famisis ${ }^{1}$
}

The main objective of this study was to provide an extensive isokinetic profile of the hip joint in youth soccer players, where the literature is limited. Additionally, this study investigated the effect of age on isokinetic peak torque values of hip abductor and adductor muscles and on reciprocal muscle group torque ratios in youth soccer players at different angular velocities (30 vs. 90o/s) and muscle actions (concentric vs. eccentric). Sixty young elite male soccer players were assigned into three equal groups $(n=20)$ : children, young adolescents and older adolescents, and performed five maximal concentric and eccentric hip-abductions and adductions at 30o/s and 90o/s. The results showed a significant increase $(p<0.05)$ in peak torque values from childhood to adolescence, with the exception of young adolescents vs. older adolescents where no differences were observed. The reciprocal ratios were not affected by age, but improved with an increase in angular velocity with the exception of the CON/ECC ratio that was higher at 30o/s. The data presented in this study provide an extensive isokinetic profile of hip abductor and adductor muscle strength in youth soccer players to assist both coaches and sports medicine professionals in strength monitoring and training.

Key words: age-effect, injury prevention, isokinetic, peak torque, reciprocal muscle group ratios.

\section{Introduction}

Concentric and eccentric muscle strength of the hip joint is an important contributing factor in the performance of several fundamental skills in soccer, such as abrupt change of direction, accelerating and decelerating (Thorborg et al., 2010, 2011a). In addition, muscular balance of the hip joint may affect athletic performance and decrease the predisposition of athletes to sport injury, reducing the incidence of groin injuries and strains of the hip joint (Kemp et al., 2013; Tyler et al., 2001). There is a notion that groin pain negatively affects the ability of an athlete to produce maximal hip adduction torque, and thereby decreases the hip adduction/abduction ratio to approximately $80 \%$, increasing consequently the risk of injury at the hip joint (Thorborg et al., 2011b). Therefore, the evaluation of hip abductors' and adductors' muscle strength as well as the implementation of reciprocal muscle group torque ratios can help coaches and sports medicine professionals, such as physicians and physiotherapists, to guide safe and effective preventive and rehabilitation protocols for groinand hip- related injuries (Kea et al., 2001; Thorborg et al., 2011a). For example, there is evidence that the hip adduction to abduction torque ratio of more than $90 \%$ constitutes a clinical milestone for an athlete striving to return to sporting activities after an adductor strain injury (Nicholas and Tyler, 2002).

It is well known that muscle strength of lower limbs increases with chronological age in trained boys (Buchanan and Vardaxis, 2009; Ioakimidis et al., 2004; Mikolajec et. al., 2012).

1 - Department of Physical Education and Sport Sciences, University of Thessaly, Karies 42100 Trikala, Greece.

2 - Department of Physical Education and Sport Sciences, Aristotle University of Thessaloniki, Thermi 57001, Greece. 
Several studies have documented increases in isokinetic (eccentric and concentric) peak torque of the knee (Gerodimos et al., 2003; Kellis et al., 2001) and ankle joint (Buchanan and Vardaxis, 2009; Gerodimos et al., 2006) in youth athletes during growth and development. At the hip joint, the few studies (Brent et al., 2013; Buchanan and Vardaxis, 2009; Quatman-Yates et al., 2013) that examined the age-related differences in isokinetic peak torque values, during the developmental ages, are limited to a concentric muscle action at a single angular velocity, in spite of the fact that eccentric muscle strength of the hip joint is an important element for success in soccer (Thorborg et al., 2010). More specifically, Brent et al. (2013) and Quatman-Yates et al. (2013) investigated the age-related differences in concentric strength of hip muscles, in a mixed sample of young male and/or female soccer and basketball players, evaluating only the hip abductor muscles.

The only study (Buchanan and Vardaxis, 2009) that assessed muscle strength of both hip abductor and adductor muscles during the developmental ages has been performed in basketball players. It is known, however, that the pattern of improvement in muscle strength may vary when examining a population with different characteristics. Soccer requires specific skills and muscle actions at different velocities during training and competition; and thus, the development of concentric and eccentric peak torque of hip abductor and adductor muscles may be different in youth soccer players compared to young athletes of other sports or to non-athletes. Therefore, it is of interest to investigate the sportspecific effect on the pattern of improvement of hip muscle strength during growth and development.

To the best of our knowledge, although previous studies have reported hip muscle strength measurements in adult soccer players (Masuda et al., 2003, 2005), there are no reports on the isokinetic strength profile of the hip joint over a range of muscle actions and angular velocities in youth athletes. Additionally, no study has examined the age-related differences in reciprocal muscle group torque ratios (conventional and functional) of the hip joint during growth and development. This is of crucial importance as conventional and functional reciprocal muscle group ratios of hip adductor and abductor muscles constitute a measure of hip joint stability and may provide information for hip function and injury risk.

Therefore, the main objective of the present investigation was to provide an extensive isokinetic profile of the hip joint in youth soccer players. Furthermore, the purpose of this study was to investigate the effect of age (children vs. young adolescents vs. older adolescents) on peak torque values of hip abductor and adductor muscles at different muscle actions (concentric vs. eccentric) and angular velocities (30o/s vs. 90o/s). The effects of age and angular velocity on conventional and functional reciprocal muscle group torque ratios at the hip joint were also investigated. The evaluation of strength of hip abductor and adductor muscles, as well as the implementation of reciprocal muscle group torque ratios (conventional and functional) can help coaches and sports medicine professionals, such as physicians and physiotherapists, to make appropriate decisions concerning performance enhancement, injury prevention/rehabilitation, and efficacy of treatment strategies.

\section{Methods}

\section{Participants}

Sixty young elite male soccer players, who trained in the talents program of the Greek Amateur Soccer Association, volunteered to participate in the present study. The participants were allocated according to their chronological age into three equal groups $(n=20)$ : children (age: $11.9 \pm 0.3 \mathrm{yrs}$; Tanner stage: 1-2; body height: 1.57 $\pm 0.08 \mathrm{~m}$; body mass: $47.0 \pm 8.6 \mathrm{~kg}$; training experience: $3.7 \pm 1.6 \mathrm{yrs}$; training frequency: $3.0 \pm$ 0.5 days/week), young adolescents (age: $15.0 \pm 0.5$ yrs; Tanner stage: 3 ; body height: $1.72 \pm 0.05 \mathrm{~m}$; body mass: $63.3 \pm 7.2 \mathrm{~kg}$; training experience: $5.8 \pm$ $2.0 \mathrm{yrs}$; training frequency: $3.7 \pm 0.7$ days/week), and older adolescents (age: $17.5 \pm 0.3$ yrs; Tanner stage: $4-5$; body height: $1.75 \pm 0.05 \mathrm{~m}$; body mass: $67.1 \pm 6.2 \mathrm{~kg}$; training experience: $8.4 \pm 2.0 \mathrm{yrs}$; training frequency: $4.9 \pm 0.6$ days/week). Before the initiation of the study, the children's parents completed a health history and a physical activity questionnaire (American College of Sports Medicine, 2013; Bar-Or, 1983). All participants were healthy and free of any illness, disease and injury of the lower limbs for at least one year prior to the study and did not report the use of any 
performance enhancing substances. The participants were engaged in conventional soccer training at least three times per week, for more than 12 months, and did not follow any specific strength and conditioning training program during the study. Additionally, the participants had no previous experience with isokinetic evaluation. Prior to the initiation of the study, the subjects and children's parents were informed about the evaluation procedure and signed an informed consent form. The research was conducted according to the ethical standards of the Declaration of Helsinki and the ethical approval was granted by the Institutional review board committee of the Department of Physical Education and Sport Sciences of the University of Thessaly.

\section{Measures}

Following orientation and completion of a medical history form, the anthropometric characteristics (body mass and body height) were assessed. Next, the participants performed a standardized 15 min warm-up that included 10 min of stationary cycling and $5 \mathrm{~min}$ of static and dynamic stretching exercises.

The isokinetic peak torque of hip abductor and hip adductor muscles was assessed using an isokinetic dynamometer (Cybex Norm ${ }^{\circledR}$, Lumex Corporation, Ronkohoma, NY). Isokinetic dynamometers have been consistently used as simple, easily applicable, and, in most cases, acceptably reliable devices for assessing lower limbs muscle strength in sports and rehabilitation settings (Claiborne et al., 2009; Kellis et al., 1999; Kemp et al., 2013). The system records the moment, angular position and velocity of the dynamometer lever arm at a sampling rate of 100 Hz. Prior to the initiation of the study, the isokinetic dynamometer was calibrated according to the instructions of the manufacturer. All tests were performed from the side-lying position, with the hip and knee, of the tested leg, extended and neutrally rotated. During the test, velcro straps were used to stabilize the trunk, waist, and thigh of the tested and non-tested legs. The resistance pad was placed to the thigh, $5 \mathrm{~cm}$ above the base of the patella (Baldon et al., 2012). The axis of rotation of the dynamometer was carefully aligned with a point representing the intersection of two lines: one line directed inferiorly from the posterosuperior iliac spine toward the knee and the other posteriorly and medially directed from the greater trochanter of the femur toward the midline of the body, as previously described by Baldon et al. (2009).

The non-tested leg was positioned at approximately $30^{\circ}$ of hip flexion in order to avoid contact with the tested leg during adduction movements (Kea et al., 2001). Only the preferred leg was tested as other studies have reported no significant bilateral differences in isokinetic peak torque of hip abductor and adductor muscles in adult soccer players (Masuda et al., 2003, 2005). Furthermore, a previous study conducted in our laboratory that was performed in young soccer players with similar characteristics (age, training status), using the same testing protocol, did not report significant differences in isokinetic peak torque of hip abductor and adductor muscles between the preferred and the non-preferred legs (Gerodimos et al., 2015).

Following positioning and stabilization on the isokinetic dynamometer, the participants performed 3 to 5 preliminary familiarization trials of each type of muscle contraction and angular velocity with low intensity not capable to induce muscle fatigue. Next, the participants completed the testing protocol consisting of five maximal concentric and eccentric hip abductions and adductions at angular velocities of $30 \% \mathrm{~s}$ and $90 \% \mathrm{~s}$ (Gerodimos et al., 2015). For all participants, the range of motion was set from $5^{\circ}$ (adduction) to $35^{\circ}$ (abduction), as previously proposed by Kea et al. (2001). Visual feedback and consistent verbal encouragement were provided during isokinetic evaluation. The eccentric and concentric tests were performed separately in randomized order. A five-minute rest was given between angular velocity and muscle action tests. The moments were corrected for the effects of gravity, and the highest torque value of 5 attempts was used for further analysis. The test-retest reliability for the assessment of hip abduction and adduction isokinetic strength, as indicated in a previous study using the same protocol, was moderate to high $(\mathrm{ICC}=0.71-0.92)$ in youth soccer players (Gerodimos et al., 2015).

The absolute peak torque $(\mathrm{N} \cdot \mathrm{m})$ of hip abductor and adductor muscles was evaluated, and the relative peak torque per unit of body mass was calculated ( $\mathrm{Nm}$ of peak torque per kilograms of body mass). The conventional 
$(\mathrm{CON} / \mathrm{CON}$ and $\mathrm{ECC} / \mathrm{ECC})$ and functional (ECC/CON and CON/ECC) hip abduction (HAB) to hip adduction (HAD) torque ratios were also calculated (Gerodimos et al., 2015). The conventional $\mathrm{HAB} / \mathrm{HAD}$ torque ratios were calculated by: a) dividing the maximal concentric torque of $\mathrm{HAB}$ (CONHAB) by the maximal concentric torque of HAD (CONHAD) and multiplying by 100 ; or $\mathrm{b}$ ) dividing the maximal eccentric torque of HAB (ЕСС eccentric torque of HAD (ECCHAD) and multiplying by 100 . The functional $\mathrm{HAB} / \mathrm{HAD}$ torque ratios were calculated by dividing the maximal eccentric torque of HAB (ECC maximal concentric torque of HAD (CONHAD) and multiplying by 100 or vice versa (Gerodimos et al., 2015).

\section{Design and Procedures}

A week before the initiation of the study, the participants were informed about the testing procedures and were familiarized with isokinetic evaluation. On the same day, the biological age was assessed by a pediatrician using Tanner's sexual maturation stages (pubic hair development) (Tanner and Whitehouse, 1976). Furthermore, the "leg preference" was determined by asking the participant "Which leg do you use to kick a ball?". Following the familiarization session, the participants reported to the laboratory to perform isokinetic testing. Participants were instructed to follow their normal diet for two days before testing, to abstain from any caffeine, tobacco and alcohol consumption for at least $24 \mathrm{~h}$ before testing, to avoid intense exercise activity for $48 \mathrm{~h}$ prior to testing, and to have sufficient rest the night before testing. All measures were performed at the same time of the day (10-12 a.m.) to prevent potential confounding effects of daily biorhythms.

\section{Statistical analysis}

All data are presented as means $\pm S D$, and were analysed using SPSS 15.0 (Illinois, USA). The normality of data was examined using the Shapiro-Wilk test. For each muscle group, threeway ANOVAs (age $x$ muscle action $x$ angular velocity, $3 \times 2 \times 2$ ) with repeated measures on "angular velocity" and "muscle action" factors were used to examine the effects of angular velocity, type of muscle action and age on absolute and relative isokinetic peak torque. Twoway ANOVAs (age $x$ angular velocity, $3 \times 2$ ) with repeated measures on "angular velocity" factor were used to examine the effects of angular velocity and age on conventional and functional reciprocal muscle group torque ratios. Significant ANOVAs were followed by Sidak pair-wise comparisons to locate the significantly different means. The level of significance was set at $p<0.05$. Furthermore, the effect sizes (ES) were calculated using Cohen's d ( $\mathrm{d}=$ difference between means : pooled SD). The small, medium, and large effects would be reflected in values greater than 0.20 , 0.50 and 0.80 , respectively, as previously described by Cohen (1972).

\section{Results}

\section{Peak torque}

ANOVA results indicated significant "age", "muscle action" and "angular velocity" $(p<$ $0.001)$ main effects and a non-significant threeway interaction $(p>0.05)$. Pair-wise comparisons within age revealed that children exhibited lower $(p<0.001)$ absolute peak isokinetic torque than young adolescents and older adolescents, whereas young adolescents vs. older adolescents did not differ in concentric and eccentric peak torque of hip abductor and hip adductor muscles irrespective of angular velocity and muscle action. The peak torque values were significantly $(p<$ 0.001 ) higher at $30 \% \mathrm{~s}$ compared to $90 \% \mathrm{~s}$ for both hip abductor and adductor muscles irrespective of muscle action and age. Additionally, peak torque values during eccentric muscle action were significantly $(p<0.001)$ higher compared to those observed during concentric muscle action regardless of angular velocity and age.

Next, the absolute peak torque values were adjusted per unit of body mass (relative torque values). Significant main effects of "age", "muscle action" and "angular velocity" $(p<0.001)$ were observed in relative peak torque of hip abductor and adductor muscles, but nonsignificant three-way interaction $(p>0.05)$. Children exhibited lower $(p<0.001)$ relative peak torque values than young and older adolescents, whereas no differences were observed between young and older adolescents irrespective of angular velocity and muscle action (Figure 1). Furthermore, the relative torque values were significantly $(p<0.001)$ higher at slow angular velocity compared to fast angular velocity irrespective of muscle action and age, and 
significantly $(p<0.001)$ higher during eccentric muscle action compared to concentric muscle action within all angular velocities and agegroups.

The absolute and relative peak torque values at each age, angular velocity and muscle action for hip abductor and adductor muscles are presented in Table 1.

\section{Reciprocal muscle group torque ratios}

ANOVA results demonstrated no significant effect of "age" on conventional and on functional reciprocal muscle group torque ratios at both slow and fast angular velocities (Table 2).

However, there was a significant "angular velocity" main effect on both conventional $(p<0.05)$ and functional $(p<0.05-0.001)$ muscle group torque ratios in youth soccer players. Conventional (CON/CON and ECC/ECC) ratio values were significantly lower at slow angular velocity compared to those observed at fast angular velocity within all age groups $(p<0.05$; Figure 2). ECC/CON functional ratio values were significantly lower at slow angular velocity compared to those observed at fast angular velocity, whereas, CON/ECC functional ratio values were significantly higher at $30 \% / \mathrm{s} \mathrm{compared}$ to those reported at $90 \% \mathrm{~s}$ regardless of age $(p<$ 0.05 ; Figure 2).

Table 1

Eccentric and concentric absolute $(\mathrm{N} \cdot \mathrm{m})$ and relative $(\mathrm{N} \cdot \mathrm{m} / \mathrm{kg})$ isokinetic peak torque values of hip abductor and adductor muscles at 30\% and 90\% angular velocities in different age-groups (mean $\pm S D)$. Adductors

\begin{tabular}{|c|c|c|c|c|c|}
\hline Velocity & Contraction & & Children & Young adolescents & Older adolescents \\
\hline \multirow[b]{2}{*}{$30 \% / s$} & Concentric & Absolute & $55.25 \pm 13.91$ & $97.15 \pm 22.68$ & $112.25 \pm 22.50$ \\
\hline & Eccentric & Relative & $1.20 \pm 0.30$ & $1.52 \pm 0.25$ & $1.68 \pm 0.34$ \\
\hline \multirow{6}{*}{$90 \% / s$} & & Absolute & $66.00 \pm 12.59$ & $115.75 \pm 25.25$ & $129.10 \pm 24.18$ \\
\hline & & Relative & $1.42 \pm 0.22$ & $1.82 \pm 0.30$ & $1.93 \pm 0.36$ \\
\hline & Concentric & Absolute & $45.50 \pm 11.56$ & $80.40 \pm 24.28$ & $95.45 \pm 21.75$ \\
\hline & Eccentric & Relative & $0.99 \pm 0.28$ & $1.26 \pm 0.33$ & $1.43 \pm 0.32$ \\
\hline & & Absolute & $62.25 \pm 13.02$ & $107.20 \pm 24.88$ & $111.40 \pm 18.16$ \\
\hline & & Relative & $1.34 \pm 0.24$ & $1.69 \pm 0.29$ & $1.67 \pm 0.29$ \\
\hline \multicolumn{6}{|c|}{ Abductors } \\
\hline Velocity & Contraction & & Children & Young adolescents & Older adolescents \\
\hline \multirow{3}{*}{$30 \% / \mathrm{s}$} & Concentric & Absolute & $65.70 \pm 16.06$ & $121.15 \pm 29.31$ & $132.0 \pm 25.56$ \\
\hline & Eccentric & Relative & $1.40 \pm 0.21$ & $1.90 \pm 0.30$ & $1.97 \pm 0.39$ \\
\hline & & Absolute & $65.35 \pm 14.95$ & $120.25 \pm 26.89$ & $137.05 \pm 24.74$ \\
\hline \multirow{5}{*}{$90 \% / \mathrm{s}$} & & Relative & $1.40 \pm 0.24$ & $1.89 \pm 0.30$ & $2.05 \pm 0.37$ \\
\hline & Concentric & Absolute & $57.60 \pm 15.34$ & $105.20 \pm 22.63$ & $111.30 \pm 21.27$ \\
\hline & Eccentric & Relative & $1.22 \pm 0.19$ & $1.65 \pm 0.21$ & $1.66 \pm 0.28$ \\
\hline & & Absolute & $62.45 \pm 16.18$ & $118.50 \pm 28.53$ & $124.85 \pm 20.32$ \\
\hline & & Relative & $1.33 \pm 0.26$ & $1.86 \pm 0.32$ & $1.87 \pm 0.30$ \\
\hline
\end{tabular}




\begin{tabular}{|c|c|c|c|c|}
\hline \multicolumn{5}{|c|}{$\begin{array}{c}\text { Table } 2 \\
\text { Hip abductor/adductor ratios }(\%) \text { at 30\% and 90\% angular velocities } \\
\text { in different age-groups (mean } \pm \text { SD). }\end{array}$} \\
\hline Ratios & Velocity & Children & Young adolescents & Older adolescents \\
\hline CONHAB/CONHAD & $\begin{array}{l}30 \% / \mathrm{s} \\
90 \% / \mathrm{s}\end{array}$ & $\begin{array}{l}121.72 \pm 29.38 \\
132.48 \pm 41.56\end{array}$ & $\begin{array}{l}126.07 \pm 19.43 \\
138.98 \pm 38.90\end{array}$ & $\begin{array}{l}118.38 \pm 15.39 \\
118.71 \pm 16.40\end{array}$ \\
\hline ЕССНАв/ЕССНАD & $\begin{array}{l}30 \% / \mathrm{s} \\
90 \% / \mathrm{s}\end{array}$ & $\begin{array}{l}99.12 \pm 14.64 \\
99.99 \pm 14.53\end{array}$ & $\begin{array}{l}105.50 \pm 19.91 \\
112.68 \pm 24.20\end{array}$ & $\begin{array}{l}107.03 \pm 11.29 \\
113.03 \pm 15.32\end{array}$ \\
\hline ECС HAB/CONHAD & $\begin{array}{l}30 \% / \mathrm{s} \\
90 \% / \mathrm{s}\end{array}$ & $\begin{array}{l}121.90 \pm 31.66 \\
144.29 \pm 47.34\end{array}$ & $\begin{array}{l}126.23 \pm 24.39 \\
158.26 \pm 53.81\end{array}$ & $\begin{array}{l}123.63 \pm 19.08 \\
134.42 \pm 22.67\end{array}$ \\
\hline CONHAB/ECCHAD & $\begin{array}{l}30 \% / \mathrm{s} \\
90 \% / \mathrm{s}\end{array}$ & $\begin{array}{l}99.40 \pm 14.54 \\
92.29 \pm 14.66\end{array}$ & $\begin{array}{c}105.72 \pm 18.00 \\
99.96 \pm 17.91\end{array}$ & $\begin{array}{l}102.95 \pm 14.60 \\
100.42 \pm 15.09\end{array}$ \\
\hline $\begin{array}{r}\text { CONHAB/CONHAD: } c \\
\text { abduction/eccentric } h \\
\mathrm{CO}\end{array}$ & $\begin{array}{l}\text { hip } a b d u \\
\text { Oon, } E C C\end{array}$ & $\begin{array}{l}\text { Oncentric hip } \\
\text { NHAD: eccentr } \\
\text { hip abduction }\end{array}$ & $\begin{array}{l}\text { ction, } E C C_{H A B} / E C \\
\text { abduction/conce } \\
\text { ntric hip adductio }\end{array}$ & $\begin{array}{l}\text { ic : eccentric hip } \\
\text { ric hip adduction, }\end{array}$ \\
\hline
\end{tabular}

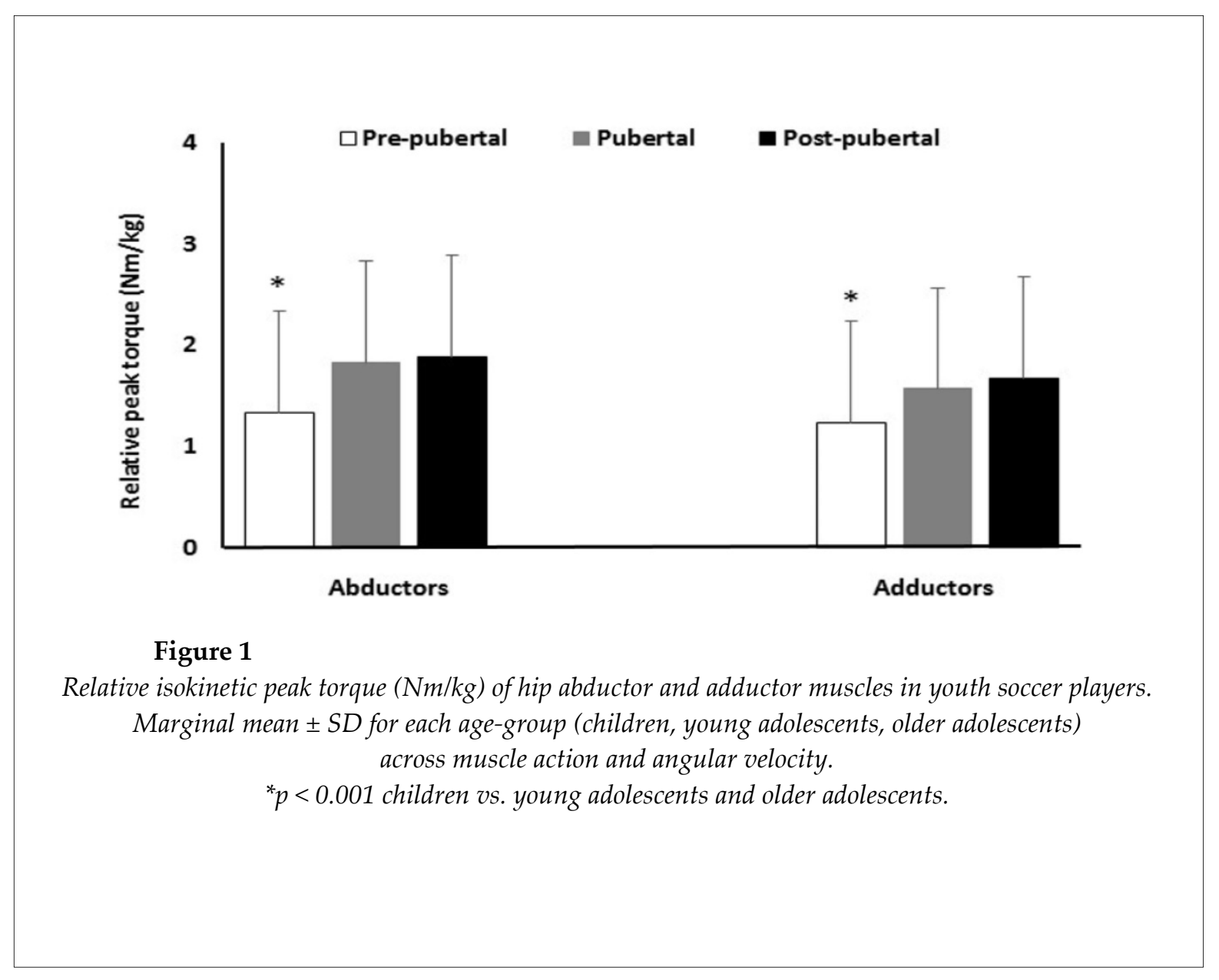




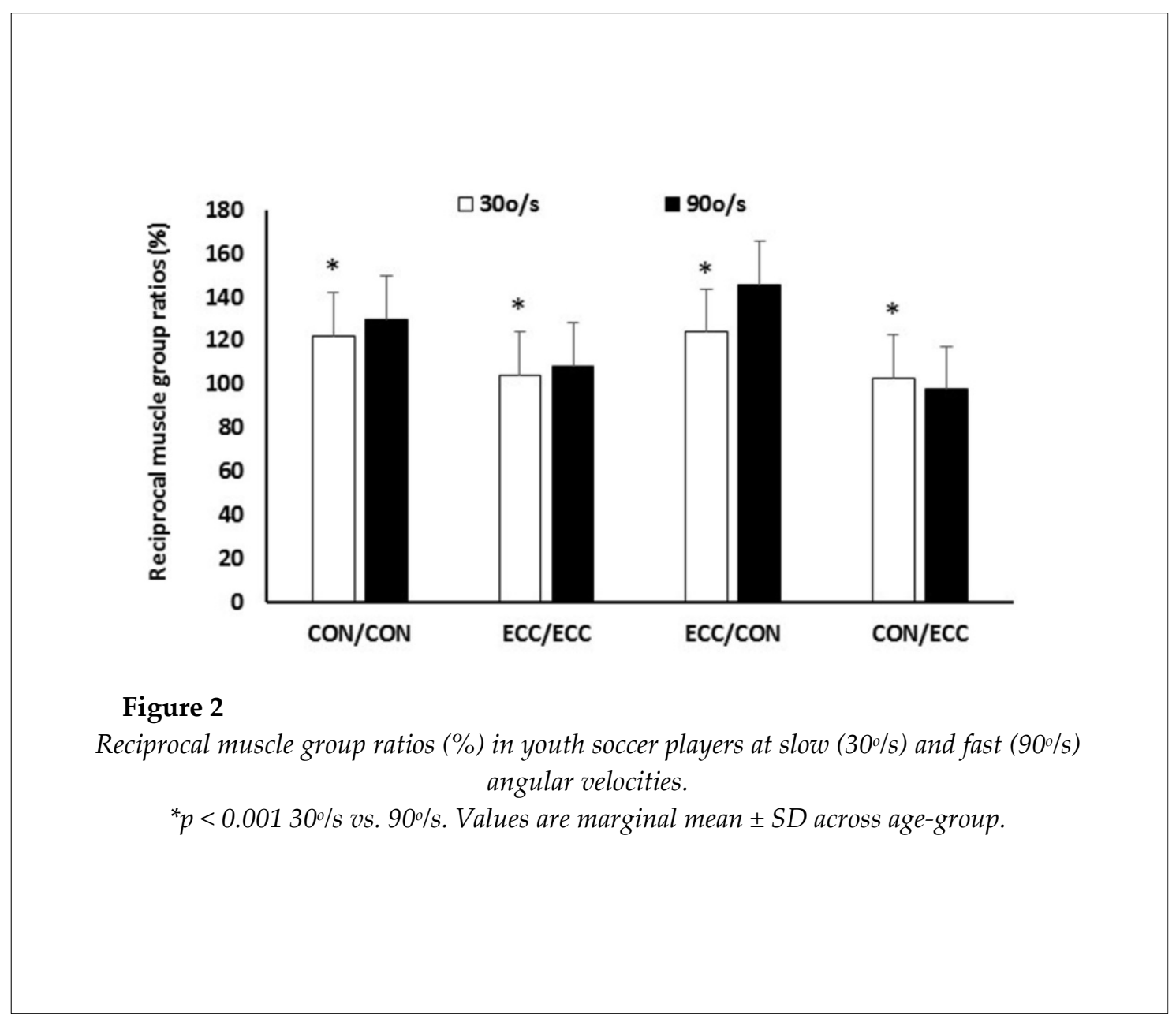

\section{Discussion}

The main findings of this study are that both absolute and relative isokinetic peak torque values of hip abductor and adductor muscles increased with chronological age in youth soccer players, with the exception of young adolescents vs. older adolescents. We also found that absolute and relative peak torque values of hip abductor and adductor muscles were significantly higher at slow angular velocity and during eccentric muscle activation regardless of age and muscle action or angular velocity. Our results indicated a nonsignificant effect of chronological age on conventional and functional muscle group torque ratios, but significant angular velocity-related effect. Specifically, CON/CON, ECC/ECC and ECC/CON values increased as angular velocity increased, while CON/ECC decreased.

Peak torque

To the best of our knowledge this is the first study that assessed the isokinetic muscle strength of hip abductor and adductor muscles in youth soccer players from childhood to adolescence over a range of angular velocities and muscle actions. There are two studies (Brent et al., 2013; Quatman-Yates et al., 2013) that evaluated isokinetic peak torque of hip abductor muscles in a mixed sample of youth soccer and basketball players using, however, a different testing protocol to that of the present investigation (standing position and angular velocity of $120 \mathrm{o} / \mathrm{s}$ in previous studies vs. side-lying position and angular velocities of $30 \mathrm{o} / \mathrm{s}$ and $90 \mathrm{o} / \mathrm{s}$ in the present study), thus comparisons with the results of the present study are not possible.

The only study (Buchanan and Vardaxis, 2009) that used a similar protocol to that of the present investigation, has been performed in youth basketball players. The authors reported higher values (by approximately 10.8-25.8\%) for both absolute and relative strength for hip abductors and by approximately $16.9-30 \%$ lower values for hip adductors compared to those in 
pre-pubertal soccer players in the present study. These differences may be possibly explained by training stimuli exerted in basketball and soccer that potentially affect the development of concentric and eccentric peak torque of hip abductor and adductor muscles. Muscle strength, especially, of hip abductor muscles is an important element in the performance of several fundamental skills in basketball, such as sliding (Inaba et al., 2013). More specifically, the main role of hip abductor muscle strength, during sliding, is to accelerate the center of mass laterally in the earlier phase of the movement and to keep the trunk upright (Inaba et al., 2013). Whereas in soccer, strength, especially of hip adductor muscles, is an important physical attribute for different skills such as kicking, accelerating and sudden change of direction (Jensen et al., 2014; Thorborg et al., 2011a); and therefore, changes in strength of hip adductor muscles may affect soccer players' performance. The physiological attributes of sport are important contributing factors that influence the development of hip abductor and adductor muscle strength, and as a result the interpretation of reciprocal muscle group torque ratios. Unfortunately, the authors of the above mentioned study (Buchanan and Vardaxis, 2009) did not present ratio values at the hip joint, thus comparisons with the results of the present study were not possible.

Previous studies that have evaluated the isokinetic peak torque of hip abductor and adductor muscles presented large discrepancies in peak torque values (Sugimoto et al., 2014). In the present study, isokinetic peak torque values of hip adductor muscles were lower (approximately 4$31 \%$ depending on the testing protocol used) than the respective values of hip abductor muscles. In the same context, previous studies that presented an isokinetic strength profile of hip abductor and adductor muscles in youth soccer (Gerodimos et al., 2015) or basketball (Buchanan and Vardaxis, 2009) players have also reported greater abductor strength values. On the other hand, other studies have reported higher adductor than abductor strength values (Masuda et al., 2003, 2005). There is evidence that the presence or the absence of gravity correction may account for these large discrepancies in the isokinetic peak torque values of hip abductor and adductor muscles amongst studies (Sugimoto et al., 2014). Indeed, the absence of gravity correction could artificially inflate the hip adduction values and result in a reduction of hip abduction values (Sugimoto et al., 2014). More specifically, Sugimoto et al. (2014) found $28-32 \%$ peak torque differences in hip abduction and adduction values with and without gravity correction. It should be mentioned that the majority of previous studies that reported greater peak torque values at hip adductor muscles did not compensate for gravity correction (Sugimoto et al., 2014). However, there are some studies (Baldon et al., 2009; Poulmedis, 1985) that actually compensated for gravity correction, but reported greater peak torque values at hip adductor muscles. It seems, therefore, that the inclusion or exclusion of gravity correction is an important factor that may have a significant impact on isokinetic peak torque values of hip abductor and adductor muscles, and thus, it should be taken into consideration in future studies (Sugimoto et al., 2014).

We also found that isokinetic peak torque values of hip abductor and adductor muscles increased with chronological age irrespective of muscle action, angular velocity and leg preference. The gradually increasing values in isokinetic peak torque of hip abductor and adductor muscles from childhood to adolescence observed in this study, are in accordance with previous reports demonstrating an age-related increase in peak torque of the hip (Brent et al., 2013; Buchanan and Vardaxis, 2009), knee (Gerodimos et al., 2003; Kellis et al., 2001), ankle (Gerodimos et al., 2006) and shoulder joint (Dos Santos Andrade et al., 2013) in trained boys during growth and development. Endocrine changes, neural maturation, and myelination of nerve fibers that occur during growth and development, as well as the fact that pubertal boys can voluntary activate a higher percentage of available motor units may have contributed to the age-related increases in isokinetic peak torque values (Froberg and Lammert, 1996; Ramsey et al., 1990). Furthermore, our results support earlier findings (Gerodimos et al., 2003, 2006) that absolute and relative peak torque values are significantly higher at low compared to high angular velocities, and significantly higher during eccentric compared to concentric contraction. Reciprocal muscle group torque ratios

The asymmetry in strength of reciprocal 
muscle groups may increase the risk for muscle injury (Kellis et al., 2000; Thorborg et al., 2010, 2011a). The abduction-to-adduction (Sugimoto et al., 2014) torque ratios are used in sports medicine to monitor potential groin- and hip-related injuries, and to assess the strength profile of the hip joint. Increased strength of hip adductor muscles and the $\mathrm{AD} / \mathrm{AB}$ torque ratio of more than $90 \%$ following a rehabilitation training program, have been reported to prevent groin- and hiprelated injuries as well as anterior cruciate ligament injuries (Nicholas and Tyler, 2002). This is the first study, to the best of our knowledge, that presents values for conventional and functional reciprocal muscle group torque ratios of hip abductor and adductor muscles in youth athletes throughout the developmental years. Young adolescents in this study exhibited conventional and functional reciprocal muscle group torque ratio values that are relatively similar to those previously reported (Gerodimos et al., 2015) in adolescent soccer players (99.9$158.2 \%$ in this study vs. $98.2-147.1 \%$ in the previous study).

Our results showed non age-related differences for both conventional and functional muscle group torque ratios, suggesting uniformity in the development of peak isokinetic torque of hip abductor and adductor muscles in youth soccer players. These findings support previous results in youth athletes reporting no significant age effect on conventional and functional muscle group torque ratios of the knee, ankle and shoulder joint in basketball or handball players (Dos Santos Andrade et al., 2013; Gerodimos et al., 2003, 2006).

The angular velocity of the movement is an important factor that could potentially affect the reciprocal muscle group torque ratio values. Indeed, our findings indicated significant differences in reciprocal ratio values between the two angular velocities (30o/s vs. $90 \mathrm{o} / \mathrm{s})$. This effect, however, differed depending on the method used to estimate reciprocal ratios (conventional vs. functional). More specifically, conventional ratios as well as the ECC/CON functional ratio increased with increasing angular velocity, which is in line with previous results at the knee joint (Aagaard et al., 1998; Gerodimos et al., 2003). In contrast, the CON/ECC functional ratio decreased with increasing angular velocity. This is in agreement with other studies that examined the angular velocity effect on functional torque ratios of the knee and ankle joint (Aagaard et al., 1998; Gerodimos et al., 2003, 2006) and should be attributed to differences in isokinetic torquevelocity characteristics between eccentric and concentric muscle actions. There is evidence that as angular velocity of the movement increases, the maximum torque generation capacity of the antagonist musculature increases, especially when the antagonists lengthen and the agonist muscles produce concentric force during the movement. This may negatively affect hip joint stabilization, especially if one considers the high magnitude forces exerted due to the lengthening action of the hip adductor muscles.

In conclusion, the data presented in this study provide an extensive profile of hip abductor and adductor muscle strength in youth soccer players that may assist both coaches and sports medicine professionals such as physicians and physiotherapists in the development of training programs for performance enhancement and rehabilitation. The isokinetic peak torque of hip abductor and adductor muscles in youth soccer players improved independent of the increase in body mass. Eccentric peak torque values were greater than the respective concentric values regardless of age and angular velocity. Furthermore, as angular velocity increased both concentric and eccentric peak torque values decreased. The functional and conventional reciprocal muscle group ratios were not affected by age. We also found that the CON/ECC functional muscle group torque ratio decreased as angular velocity increased, while conventional and $\mathrm{ECC} / \mathrm{CON}$ functional ratio values increased.

\section{Acknowledgements}

We would like to thank the participants of the study for volunteering their time. No external financial support was received for this research. 


\section{References}

Aagaard P, Simonsen EB, Magnusson SP, Larsson B, Dyhre-Poulsen P. A new concept for isokinetic hamstring:quadriceps muscle strength ratio. Am J Sport Med, 1998; 26: 231-237

American College of Sports Medicine. ACSM's Guidelines for Exercise Testing and Prescription. 9th ed. Philadelphia: Lippincott Williams \& Wilkins; 2013

Baldon RM, Lobato DFM, Carvalho LP, Wun PYL, Presotti CV, Serrão FV. Relationships between eccentric hip isokinetic torque and functional performance. J Sport Rehab, 2012; 21: 26-33

Baldon RM, Nakagawa TH, Muniz TB, Amorim CF, Maciel CD, Serrão FV. Eccentric hip muscle function in females with and without patellofemoral pain syndrome. J Athl Train, 2009; 44: 490-496

Bar-Or O, ed. Pediatric Sports Medicine for the Practioner: From Physiologic Principles to Clinical Application. New York: Springer-Verlag; 1983

Brent JL, Myer GD, Ford KR, Paterno MV, Hewett TE. The effect of sex and age on isokinetic hip-abduction torques. J Sport Rehab, 2013; 22: 41-46

Buchanan PA, Vardaxis VG. Lower-extremity strength profiles and gender-based classification of basketball players ages 9-22 years. J Strength Condit Res, 2009; 23: 406-419

Claiborne TL, Timmons MK, Pincivero DM. Test-retest reliability of cardinal plane isokinetic hip torque and EMG. J Electromyogr Kinesiol, 2009; 19: e345-352

Cohen J. Statistical power analysis for the behavioral sciences. New York: American Elsevier; 1972

Dos Santos Andrade M, Barbosa de Lira CA, Vancini RL, Aparecido de Almeida A, Benedito-Silva AA, da Silva AC. Profiling the isokinetic shoulder rotator muscle strength in 13- to 36-year-old male and female handball players. Phys Ther Sport, 2013; 14: 246-252

Froberg K, Lammert O. Development of muscle strength during childhood. In: Bar-Or O, eds. The Child and Adolescent Athlete: The Encyclopedia of Sports Medicine. Oxford: Blackwell Science, 25-40; 1996

Gerodimos V, Karatrantou K, Paschalis V, Zafeiridis A, Katsareli E, Bilios P, Kellis S. Reliability of concentric and eccentric strength of hip abductors and adductors muscles in young soccer players. Biol Sport, 2015; 32: 351-356

Gerodimos V, Mandou V, Zafeiridis A, Ioakimidis P, Stavropoulos N, Kellis S. Isokinetic peak torque and hamstring/quadriceps ratios in young basketball players: Effects of age, velocity, and contraction mode. J Sports Med Phys Fitness, 2003; 43: 444-452

Gerodimos V, Manou V, Stavropoulos N, Kellis E, Kellis S. Agonist and antagonist strength of ankle musculature in basketball players aged 12 to 17 years. Isokinet Exerc Sci, 2006; 14: 81-89

Inaba Y, Yoshioka S, Iida Y, Hay DC, Fukashiro S. A biomechanical study of side steps at different distances. J Appl Biomech, 2013; 29: 336-345

Ioakimidis P, Gerodimos V, Kellis E, Alexandris N, Kellis S. Combined effects of age and maturation on maximum isometric leg press strength in young basketball players. J Sports Med Phys Fitness, 2004; 44: 389-397

Jensen J, Bandholm T, Holmich P, Thorborg K. Acute and sub-acute effects of repetitive kicking on hip adduction torque in injury-free elite youth soccer players. J Sports Sci, 2014; 32: 1357-1364

Kea J, Kramer J, Forwell L, Birmingham T. Hip abduction-adduction strength and one-leg hop tests: testretest reliability and relationship to function in elite ice hockey players. J Orthop Sports Phys Ther, 2001; 31: $446-455$

Kellis E, Kellis S, Gerodimos V, Manou V. Reliability of isokinetic concentric and eccentric muscle strength in young soccer players. Pediatr Exerc Sci, 1999; 11: 218-228

Kellis S, Gerodimos V, Kellis E, Manou V. Bilateral isokinetic concentric and eccentric strength profiles of the knee extensors and flexors in young soccer players. Isokinet Exerc Sci, 2001; 9: 31-39 
Kellis S, Kellis E, Manou V, Gerodimos V. Prediction of knee extensor and flexor isokinetic strength in young male soccer players. Journal Orthop Sports Phys Ther, 2000; 30: 693-701

Kemp JL, Schache AG, Makdissi M, Sims KJ, Crossley KM. Greater understanding of normal hip physical function may guide clinicians in providing targeted rehabilitation programmes. J Sci Med Sport, 2013; 16: $292-296$

Masuda K, Kikuhara N, Demura S, Katsouta S, Yamanaka K. Relationship between muscle strength in various isokinetic movements and kick performance among soccer players. J Sports Med Phys Fitness, 2005; 45: 44-52

Masuda K, Kikuhara N, Takahashi H, Yamanaka K. The relationship between muscle cross-sectional area and strength in various isokinetic movements among soccer players. J Sports Sci, 2003; 21: 851-858

Mikolajec K, Waskiewicz, Z, Maszczyk A, Bacik B, Kurek P, Zajac, A. Effects of stretching and strength exercises on speed and power abilities in male basketball players. IES, 2012; 20(1): 61-69

Nicholas SJ, Tyler TF. Adductor muscle strains in sport. Sports Med, 2002; 32: 339-344

Poulmedis P. Isokinetic maximal torque power of greek elite soccer players. J Orthop Sports Phys Ther, 1985; 6: 293-295

Quatman-Yates CC, Myer GD, Ford KR, Paterno MV, Hewett TE. A longitudinal evaluation of maturational effects on lower extremity strength in female adolescent athletes. Pediatr Phys Ther, 2013; 25: 271-276

Ramsey JA, Blimkie CJR, Smith K, Garner S, MacDougall JD, Sale DG. Strength training effects in prepubescent boys. Med Sci Sports Exerc, 1990; 22: 606-614

Sugimoto D, Mattacola CG, Mullineaux DR, Palmer TG, Hewett TE. Comparison of isokinetic hip abduction and adduction peak torques and ratio between sexes. Clin J Sport Med, 2014; 24: 422-428

Tanner JM, Whitehouse RH. Clinical longitudinal standards for height, weight, height velocity, weight velocity, and stages of puberty. Arch Dis Child, 1976; 51: 170-179

Thorborg K, Couppe C, Petersen J, Magnusson SP, Holmich P. Eccentric hip adduction and abduction strength in elite soccer players and matched controls: a cross-sectional study. Br J Sports Med, 2011; 45: $10-13$

Thorborg K, Petersen J, Magnusson SP, Holmich P. Clinical assessment of hip strength using a handheld dynamometer is reliable. Scand J Med Sci Sports, 2010; 20: 493-501

Thorborg K, Serner A, Petersen J, Madsen TM, Magnusson P, Hölmich P. Hip Adduction and Abduction Strength Profiles in Elite Soccer Players: Implications for Clinical Evaluation of Hip Adductor Muscle Recovery After Injury. Am J Sports Med, 2011; 39: 121-126

Tyler TF, Nicholas SJ, Campbell RJ, McHugh MP. The association of hip strength and flexibility with the incidence of adductor muscle strains in professional ice hockey players. Am J Sports Med, 2001; 29: 124128

\section{Corresponding author:}

\section{Konstantina Karatrantou,}

Department of Physical Education and Sport Sciences,

University of Thessaly, Karies 42100 Trikala, Greece.

E-mail: kokaratr@pe.uth.gr.

Tel: +302431047005 .

Fax: +302431047042 . 